\title{
The future of capsule endoscopy in clinical practice: from diagnostic to therapeutic experimental prototype capsules
}

\author{
Michael D. Vasilakakis ${ }^{1}$, Anastasios Koulaouzidis ${ }^{2,3}$, Wojciech Marlicz ${ }^{4}$, Dimitris K. Iakovidis ${ }^{1}$ \\ ${ }^{1}$ Department of Computer Science and Biomedical Informatics, University of Thessaly, Lamia, Greece \\ ${ }^{2}$ Endoscopy Unit, The Royal Infirmary of Edinburgh, Edinburgh, Scotland \\ ${ }^{3}$ Department of Clinical Sciences, Lund University, Malmö, Sweden \\ ${ }^{4}$ Department of Gastroenterology, Pomeranian Medical University, Szczecin, Poland
}

Gastroenterology Rev 2020; 15 (3): 179-193

DOI: https://doi.org/10.5114/pg.2019.87528

Key words: therapeutic capsule endoscopy, diagnostic yield, detection, algorithm, engineering, drug delivery.

Address for correspondence: Michael D. Vasilakakis, Department of Computer Science and Biomedical Informatics, University of Thessaly, Papasiopoulou 2-4, Lamia, Greece, e-mail: vasilaka@uth.gr

\begin{abstract}
Capsule endoscopy (CE) is indicated as a first-line clinical examination for the detection of small-bowel pathology, and there is an ever-growing drive for it to become a method for the screening of the entire gastrointestinal tract (GI). Although CE's main function is diagnosis, the research for therapeutic capabilities has intensified to make therapeutic capsule endoscopy (TCE) a target within reach. This manuscript presents the research evolution of CE and TCE through the last 5 years and describes notable problems, as well as clinical and technological challenges to overcome. This review also reports the state-of-the-art of capsule devices with a focus on CE research prototypes promising an enhanced diagnostic yield (DY) and treatment. Lastly, this article provides an overview of the research progress made in software for enhancing DY by increasing the accuracy of abnormality detection and lesion localisation.
\end{abstract}

\section{Introduction}

It has been 17 years since the appearance of the first commercial capsule endoscope in 2001 [1]. Capsule endoscopy (CE) has had an impact on clinical practice by offering a minimally-invasive, and hence well tolerated, alternative to conventional endoscopy for visualisation of the entire gastrointestinal tract (GI) [2]. CE devices have a shape similar to that of a large vitamin pill, and their volume is roughly $2 \mathrm{~cm}^{3}$. Nowadays, there are five leading companies in the market of $\mathrm{CE}$, which provide diagnostic tools for non-invasive exploration of the small bowel as well as for oesophageal and colonic examinations. One of these companies, Medtronic Inc., has developed the third version of its small-bowel capsule, called the PillCam ${ }^{\text {TM }}$ SB 3 [3]. Also, it has developed the PillCam Crohn's capsule, used for the visualisation of the small bowel and the colonic mucosa for the assessment of Crohn's disease (CD), as well as the Pillcam ${ }^{\mathrm{TM}}$ COLON2 and the Pillcam ${ }^{\mathrm{TM}}$ Upper GI (UGI) for the examination of the respective parts of the Gl tract. The MC2000 and the EndoCapsule (EC-S10) are the latest versions of small-bowel capsule endoscopes manufactured by Intromedic [4] and Olympus Corporation [5], respectively. Interestingly, the CapsoCam ${ }^{\circledast}$ Plus [6] by Capsovision provides a $360^{\circ}$ side-on panoramic field view due to the four cameras placed in the centre of its body. Jinshan Science and Technology, Chongqing, China has developed the OMOM Capsule 2 [7]. Also, CheckCap has developed the C-Scan ${ }^{\circledR}$ Cap [8], which is an ingestible $X$-ray-based imaging capsule that consists of an X-ray source and detects ultra-low-dose radiation. The common significant pathologies seen in the Gl tract basically fall into a few main groups: vascular lesions, neoplasms (i.e. polyps/tumours), and inflammatory lesions - idiopathic inflammatory bowel disease (IBD) and pharmacogenetic or infectious inflammation. Furthermore, even a negative CE examination is of value in guiding further investigation and management - pooled data have shown that patients with no significant find- 
ings on CE have a much lower rate of re-bleeding (19\%) compared to those with a positive capsule (40\%) [9]. This is especially important in the significant subgroup of patients with iron deficiency anaemia (IDA), who are often referred for repeated capsule investigations with poor diagnostic yield (DY) [10, 11]. CE is useful for monitoring or detecting these pathologies of the small bowel, such as CD or bleeding, because the small bowel is predominantly inaccessible to regular endoscopes due to its long and convoluted structure.

Therefore, although the current commercial capsules offer convenience and an acceptable DY, they have several drawbacks. Traversing the GI tract in a passive manner is perhaps the most important of them; physicians are not able to interfere in the movement and/or speed of the capsule as it moves in the lumen of the Gl tract propelled by contractions. In other words, it is not possible to stop or navigate the capsule towards an area of interest, for a more thorough review. Another constraint is the limited battery life, which is insufficient for multimodal diagnosis. Various research groups have proposed different prototype solutions to overcome these drawbacks. Also, the research for the enhancement of diagnostic ability of CE devices is leading towards therapeutic CE (TCE) devices. Research for TCE has been limited because of drawbacks, such as inaccurate capsule localisation that makes drug delivery difficult in specific regions of $\mathrm{Gl}$ tract. As the number of capsule endoscopy-related trials currently exceeds 190 records in ClinicalTrials.gov registry, so the demand for new capsule-based technologies and solutions is also growing [12].

In recent years, a number of review studies on CE have been published [1, 13-17]. However, CE is an area of technology with rapid development. The contribution of this review is the presentation of the endeavours from different research groups in CE and TCE. These include state-ofthe-art capsule endoscopes and software methodologies that can contribute to enhanced DY and treatment.

\section{State-of-the-art research capsule endoscopes}

\section{Diagnostic capsule prototypes}

In this context, various research groups have proposed different prototype solutions to overcome these drawbacks. A list of representative research capsule prototypes is provided in Table I. They are also described in the following paragraphs in chronological order.

\section{Four-camera high-resolution and -throughput capsule endoscope}

The group of Jang et al. [18] has developed a capsule consisting of four Video Graphics Array (VGA) cameras. The capsule has a diameter of $12 \mathrm{~mm}$ and length of
$32 \mathrm{~mm}$, and it weighs < $4 \mathrm{~g}$. The field of view for every single camera is $120^{\circ}$, enabling the capsule to capture $360^{\circ}$ images of $640 \times 480$ pixels at a frame rate of four frames per second (fps). The capsule consists of a transmitter providing image transmission of up to $80 \mathrm{Mb} / \mathrm{s}$ to an external receiver, with power consumption at $0.8 \mathrm{~mW}$. The two batteries inside the capsule can last for $>12 \mathrm{~h}$. Also, the capsule can provide images with information of the location inside the Gl tract achieving sub-centimetre accuracy. The location of the capsule is specified measuring the signal power between the capsule and the external receiver that has eight nodes and a hub implanted on a tight vest. The location is determined to be near the node with the least channel attenuation and then specified using an adaptive selection of the nearest four nodes. In-vitro experimental results in pig intestine proved the sub-centimetre accuracy.

\section{Wireless spherical endoscopic capsule}

In the framework purposed by the group of Fontana et al. [19] a capsule with spherical shape was presented. The capsule has $26 \mathrm{~mm}$ diameter and weighs $12.70 \mathrm{~g}$. The capsule transmits images of $320 \times 320$ resolution at $1.5 \mathrm{fps}$. The battery of the capsule can be recharged up to six times through a proposed recharging circuit. A power-on circuit and a localisation module are embedded in the capsule. In order to reduce the friction during examination of the colon, which leads to the camera having insufficient angles of view, a structure combining an outer and an inner shell has been constructed. These shells have a $360^{\circ}$ of orientation and the simultaneous movement maintains the camera in the correct direction. The actuation of the capsule is based on the interaction of the integrated permanent magnet in the capsule and an external electromagnet. The capsule has been tested in an in-vitro colon simulation for image capturing.

\section{Magnetically actuated soft capsule endoscope for fine-needle aspiration biopsy}

The extraction of samples from suspicious tissue lesions through a thin and hollow needle is a method called fine-needle aspiration biopsy. The group of Son et al. [20] has proposed a magnetically actuated soft capsule endoscope (B-MASCE) that utilises the fine-needle aspiration biopsy functionality. The fine needle can penetrate deep inside the mass of a lesion, even in the case of sub-mucosal tumours, and it can improve the diagnostic yield. The B-MASCE has been manufactured to enable a rolling locomotion on the surface of the stomach and the motion of axial jabbing of the needle. A magnetic field is used for controlling and torqueing of the magnet inside the capsule endoscope. Four legs as- 
Table I. List of the state-of-the-art research capsule prototypes

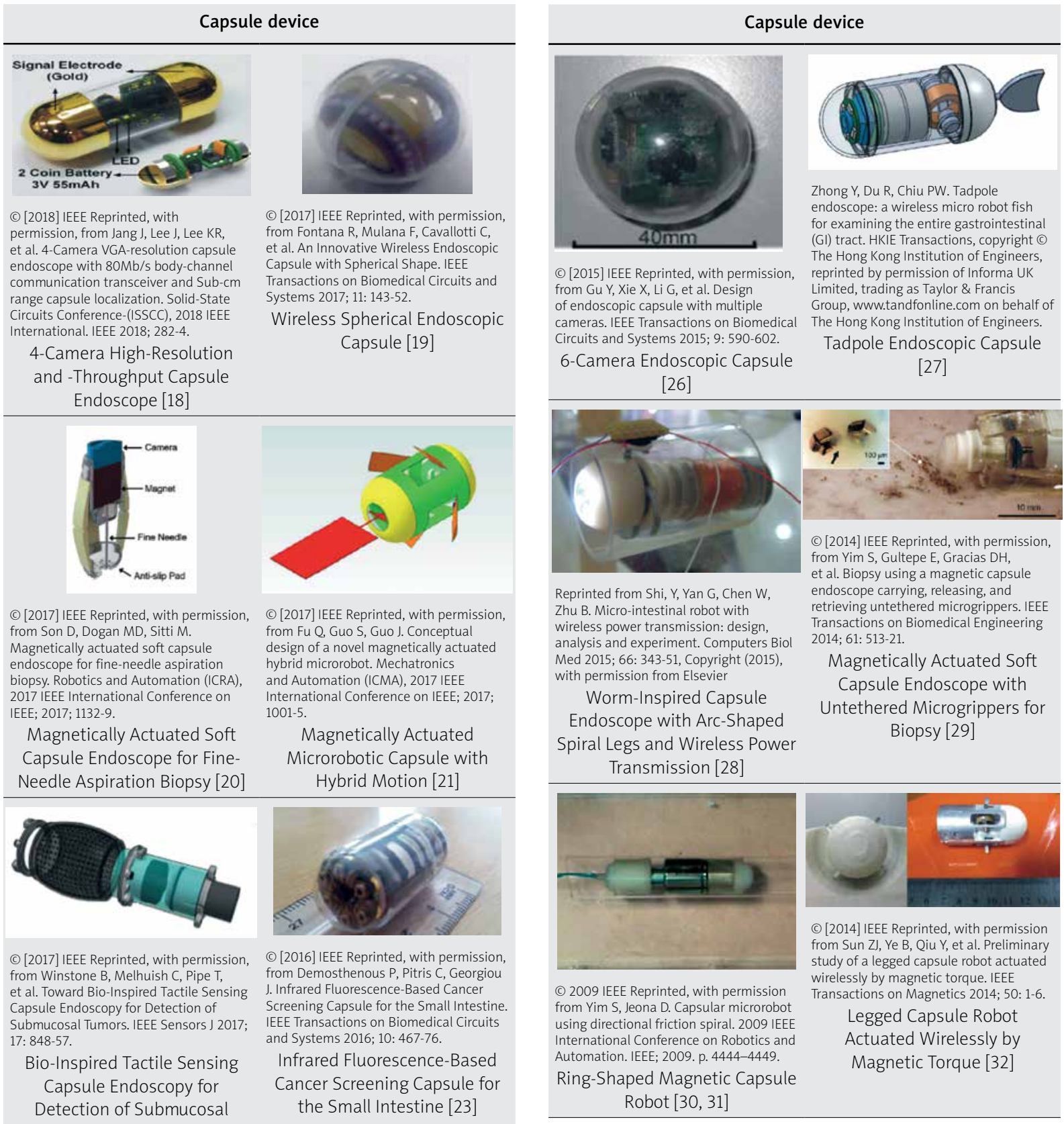


sist the guidance of the needle for the penetration in the area of the lesion. These four legs are made from soft material; thus the capsule endoscope is called "soft". The capsule is designed for the examination of the upper Gl tract, and it has diameter of $12 \mathrm{~mm}$ and length of $30 \mathrm{~mm}$. The fine needle has a length of $15 \mathrm{~mm}$ and penetration depth of $10 \mathrm{~mm}$. In vitro experiments for sampling were performed using pork fat as a GI tumour. Samples were successfully captured from the proposed capsule with fine-needle aspiration biopsy functionality.

\section{Magnetically actuated microrobotic capsule with hybrid motion}

The group of Fu et al. [21] has proposed a conceptual, magnetically actuated, hybrid micro-robot with hybrid motion, which includes screw jet, paddling, and fin motion. It is driven by an electromagnetic actuation system, which generates a rotational magnetic field and alternate magnetic field. The experimental results from simulation indicate that the micro-robot coped with the flexible motion in the pipe by adjusting the magnetic frequency. The physician has the ability to move the microrobot to accomplish functions such as endoscopy examination or drug delivery, after taking into consideration information like the position and the posture of the microrobot. The proposed dimensions of the microrobot are a diameter of $13 \mathrm{~mm}$ and length of $30 \mathrm{~mm}$.

\section{Bio-inspired tactile sensing capsule endoscopy for detection of submucosal tumours}

Inspired by human finger sensing anatomy, Winstone et al. [22] have developed a biomimetic tactile fingertip sensor, named Tactip. Tactip uses remote palpation to stimulate a tactile sensing surface that deforms when pressed against soft or hard lumps on the surface of the Gl tract. This new diagnostic method enables the enhancement of visual investigation of lesions, and it could provide further information about the structure of the lesions. A system able to classify abnormalities based on the shape, size and softness was utilized to test the sensor. Thus, information of the characteristics in different locations inside the surface of the bowel without relying on vision alone can be provided by the system. Tactip has an embedded artificial cast silicone skin, optically clear flesh like gel, a camera with $720 \times 1200$ resolution, and internal illumination using LEDs.

\section{Infrared fluorescence-based cancer screening capsule for the small intestine}

Cancer detection in early stage through infrared fluorescent labelling is a well-known technique. The group of Demosthenous et al. [23] have developed a screening capsule prototype for the detection of fluorescence emitted by very low concentrations of Indocyanine Green (ICG) fluorophores. The capsule has a diameter of $13 \mathrm{~mm}$ and length of $25 \mathrm{~mm}$. It is able to detect and record fluorescence levels for about $9 \mathrm{~h}$ via a variable sampling rate methodology that reduces the amount of redundant data collected. The fluorescence levels are stored in the internal memory of the capsule. Therefore, this capsule provides a viable general screening method for small-bowel cancer. Analysis of 8 or more hours of video for each patient is not required because the physicians can examine whether the detected fluorescence levels exceeded a predefined threshold when plotted on a chart. The proposed near infrared-based fluorometric capsule is the first of its kind, in that without external body-worn hardware and labour-intensive video analysis, early stage cancers can be detected cost-efficiently and reliably. Ex-vivo experiments of the proposed capsule were conducted using ICG-impregnated swine intestine, showing the detection and screening ability of the system at different ICG concentrations.

\section{Motor-based capsule robot powered by wireless power transmission}

Sufficient supply of power is important to support the functionalities of a capsule endoscope, such as video recording, video wireless transmission, biopsy devices, etc. However, commercially-available button batteries are not sufficient to power these functionalities for long durations. In this work, the group of Gao et al. [24] has proposed a motor-based capsule robot utilising an inchworm mechanism consisting of two expanding devices at both ends and a middle extensor for active locomotion. The capsule robot is powered by Wireless Power Transmission (WPT). WPT is based on near-field inductive coupling to supply several hundred milliwatts to the moving capsule robot and has been considered as a promising solution. The WPT system normally consists of a one-dimensional (1-D) transmitting coil that excites an alternating magnetic field and a three-dimensional (3-D) receiving coil with an inserted ferrite core that induces an electromotive force (EMF). The motor-based capsule robot has a diameter of $13 \mathrm{~mm}$ and length of $42 \mathrm{~mm}$ for exploring the intestinal tract. The frame rate of the proposed capsule is 30 frames per second with a resolution of $320 \times 240 \mathrm{dpi}$. Ex-vivo evaluation of the proposed capsule robot was performed proving that the capsule is able to navigate in a collapsed porcine intestine.

\section{Active Locomotive Intestinal Capsule Endoscope (ALICE) System}

Several mobility mechanisms for the capsule endoscope have been developed by researchers without 
achieving adequate degrees of freedom or sufficiently diverse capsule motions. The group of Lee et al. [25] have developed the Active Locomotion Intestinal Capsule Endoscope (ALICE) system with diverse mobility consists of an electro-magnetic actuation (EMA) system and capsule endoscope. The EMA system has three pairs of orthogonal uniform magnetic coils for 3-D alignment, and two pairs of gradient magnetic coils for propulsion, and it can perform complex motions of the capsule. The capsule has a tubular shape and moves through five degrees of freedom, enabling complex movements, e.g. helical movements to closely scan the inner wall of the Gl tract. The designed capsule endoscope has a diameter of $8 \mathrm{~mm}$ and length of $20 \mathrm{~mm}$. The motion of the ALICE system was evaluated in ex vivo experiments, which verified the feasibility of the ALICE system for investigation of the GI tract.

\section{Six-camera endoscopic capsule}

The random movement of the capsule and the limited visual field in the Gl tract results in a high miss rate of significant findings. For this reason, the group of Gu et al. [26] has implemented a Multiple-Camera Endoscopic Capsule (MCEC) with smart control to reduce the miss rate. Multiple cameras are employed to create a larger visual field. Instead of wirelessly transmitting the image data, it stores them in a flash memory, thus enabling a high image acquisition rate. Moreover, a low-complexity image compression algorithm is proposed, achieving a decrease in power consumption. A smart image capture control strategy based on motion information is used to control the frame rate upon random movements within the digestive tract. This prototype endoscopic capsule has six cameras and captures frames with resolution of $480 \times 480$ pixels.

\section{Tadpole endoscopic capsule}

Inspired by the movements seen in the natural world the group of Zhong et al. [27] have proposed the Tadpole Endoscope, which adopts the thunniform swimming technique of tadpoles and can propel itself through the GI tract. The Tadpole Endoscope follows the traditional capsule endoscope design, equipped with a soft tail, and contains the driving unit, camera, control- and application-specific integrated circuit transmitter, antenna, and batteries. The driving unit drives the tail to flap and generates a propulsive force. The body of the capsule has a diameter of $13 \mathrm{~mm}$ and a length of $38 \mathrm{~mm}$, of which $9 \mathrm{~mm}$ is the length of the tail. The total weight is only $6 \mathrm{~g}$, and one $1.5 \mathrm{~V}$ button battery is used to drive the circuit board and the magnetic coil. Motion instructions are sent to the circuit board via infrared communication. The maximum swimming speed is $12.5 \mathrm{~mm} / \mathrm{s}$ and the minimum turning radius is $25 \mathrm{~mm}$. The tadpole endoscope's motion was tested ex vivo in a pig stomach, where the movement of the Tadpole Endoscope and the change of direction when it reached the stomach wall were proven.

\section{Worm-inspired capsule endoscope with arc-shaped spiral legs and wireless power transmission}

Relying on Gl peristalsis, the capsule endoscope cannot actively move and treat effectively, necessitating further research into intestinal robotic devices. In order to support the locomotion in the intestinal tract capsule endoscopes are equipped with legs or paddles. However, the sharp ends of the legs have the potential to damage the intestine of the patient while navigating through it. The group of Shi et al. [28] proposed a capsule robot that mimics the movement of a worm equipped with elastic spiral legs. These arc-shaped spiral legs slide along the intestinal wall without hurting the intestine of the patient. In the body of the capsule a CMOS camera and a transmitting circuit are integrated for image acquisition. To avoid the limitation of insufficient battery duration a wireless power transmission system is used for power supply. The power supply system consists of receiving coils, a rectifier circuit, and a voltage regulator circuit. The ring-like receiving coils are designed to surround the shell and to protect the inner space of the robot. The capsule has diameter of $16 \mathrm{~mm}$ and length of $31 \mathrm{~mm}$. The locomotion and the wireless power transmission where tested in ex vivo experiments.

\section{Magnetically actuated soft capsule endoscope with untethered microgrippers for biopsy}

The group of Yim et al. [29] has developed a capsule endoscope system combining two devices for biopsy. Firstly, the system exploits the extra axial degree of freedom of the MASCE device for the functionalities of remote actuation, controlled navigation, and drug release. Secondly, the system utilises microgrippers that can be actuated autonomously at body temperature and self-fold collecting tissue samples in a highly parallel manner. The microgrippers have been optimised in order not to fold earlier than a 10-min period. This time threshold for the microgrippers to close prevents them from folding before their release from the capsule. The constructed capsule has a diameter of $18 \mathrm{~mm}$ and a length of $31.5 \mathrm{~mm}$. Ex vivo experiments were conducted, which showed that the proposed capsule is capable of the retrieval of multiple tissue samples. 


\section{Ring-shaped magnetic capsule robot}

The group of Yim et al. [30, 31] have presented a capsule robot able to move in a fluid-filled tube. The capsule is designed with three different parts. The first part is the rotating frontal part of the robot, which consists of a permanent magnet that has the shape of a ring. The second part is the linearly moving clamper that is also equipped with a permanent magnet, a clamper with ribs to support the directional movement of the capsule, and a slider. The third part is the capsule body, which consists of a stroke limiter and a small motor of a diameter $6 \mathrm{~mm}$. The mechanical arrangement of the parts provides the locomotion of the capsule. The motor rotates the frontal part. The magnetic force of the magnet of the frontal part pulls or pushes the magnet of the second part. Thus, the second part passively moves linearly and forces the movement of the capsule robot. This mechanism enables the capsule robot to achieve enough speed for the generation of appropriate propulsion inside the liquid tube.

\section{Legged capsule robot actuated wirelessly by magnetic torque}

An active locomotion legged capsule robot that can move inside the Gl tract has been proposed by Sun et al. [32]. The four legs move the capsule robot forward or backward. The activation of the legged capsule to move or to stop is achieved by magnetic torque without the dependence of a battery for power supply. The actuation of two external permanent magnets forces an internal permanent magnet to rotate. The internal permanent magnet is integrated in the capsule robot and rotates relative to the body of the robot. An internal mechanism transmits the magnetic torque of the rotating internal permanent magnet in order to activate or stop the legs.

\section{Wireless autonomous endoscope with micro-jaw forceps for biopsy}

Two types of micro-jaw mechanisms for biopsy, the slide-jaw and the sleeve-jaw, for a wirelessly powered capsule endoscope have been designed by Chen et al. [33]. The capsule robot has forceps in the front part of its body. The actuator of the capsule robot utilises the lead-screw mechanism for the stretching out and withdrawing motions of the micro-jaw. During the biopsy there are three movements that have to be executed. The first one is the extension of the micro-jaws to the sampling area. Second, the micro-jaws bite and cut off tissue. Third, the micro-jaws withdraw back in the capsule. The collected samples are stored inside the capsule. The proposed two types of micro-jaw aim to support biopsy in surgical tasks. The slide-jaw extends along a guiding slot and pull back to cut off a tissue sample. The tissue sample is kept squeezed inside the two parts of jaw. The adjustment of the forward distance of the jaw varies the amount of sampling tissue. The volume of sampling tissue is around 1 to $1.5 \mathrm{~mm}^{3}$. The sleeve-jaw forceps are inspired by traditional biopsy tools. The sleeve jaw cuts off the tissue samples utilising the pressure from the compressed spring. The sleeve jaw is used for the retrieval and storage of smaller amounts of tissue samples - about $0.5 \mathrm{~mm}^{3}$ to $1 \mathrm{~mm}^{3}$ - than the slide jaw. The cutting ability of the forceps was evaluated ex vivo using the small intestine of a pig (Table I).

\section{Therapeutic capsule prototypes}

This subsection describes the potential capsule endoscopes capable of therapeutic functionalities, such as drug delivery, in order to treat specific pathologies such as bleeding in the GI tract or Helicobacter pylori. A list of the proposed therapeutic capsule prototypes is provided in Table II. They are also described in the following paragraphs in chronological order.

\section{SonoCAIT}

The group of Steward et al. [34] has developed a proof-of-concept capsule for an ultrasound (US)-mediated targeted drug delivery (UmTDD), named SonoCAIT. The prototype capsule is suitable for drug delivery in a specific location, utilising US to release drugs and/or to increase drug uptake through sonoporation. SonoCAIT has a pill-like shape with the dimensions of $10 \mathrm{~mm}$ diameter and $30 \mathrm{~mm}$ length. An US transducer, a drug delivery channel, a vision module, and a multi-channel external tether are integrated into the capsule. The vision module consists of a CMOS camera and circuit board with four white LEDs. The camera is cylindrical with resolution of $220 \times 224$ pixels. The aim of SonoCAIT is to deliver drugs to the wall of the $\mathrm{Gl}$ tract. One example of a therapeutic preparation is drug-filled microbubbles (MBs). When these reach the target zone, they must be released in close proximity to the wall, where the drugs can then be released by US. That means the US focus and MBs have to be directed towards the same target. In vitro experiments showed that SonoCAIT achieved enhancement of drug uptake.

\section{A capsule for haemostasis utilising an inflated balloon}

An inflatable prototype capsule for haemostasis in the Gl tract based on balloon tamponade effect has 
Table II. List of state-of-the-art therapeutic capsule prototypes

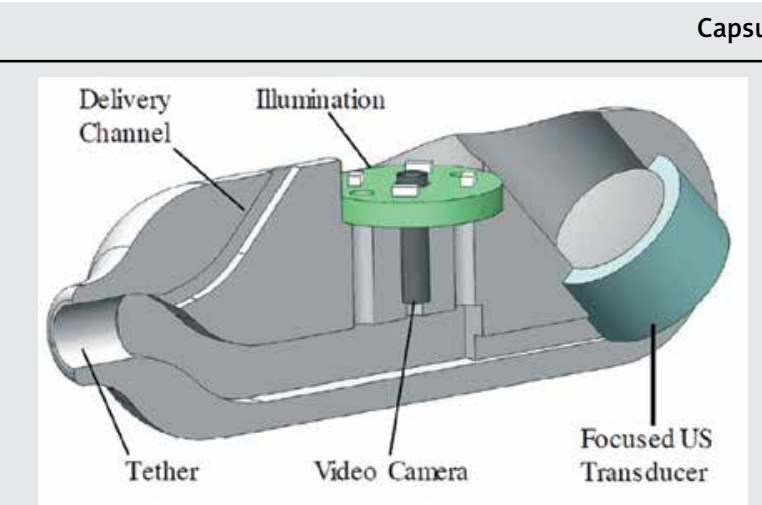

(c) [2017] IEEE Reprinted, with permission, from Stewart F, Newton IP, Näthke I, et al. Development of a therapeutic capsule endoscope for treatment in the gastrointestinal tract: Bench testing to translational trial. Ultrasonics Symposium (IUS), 2017 IEEE International. IEEE 2017; 1-4.

\section{SonoCAIT [34]}

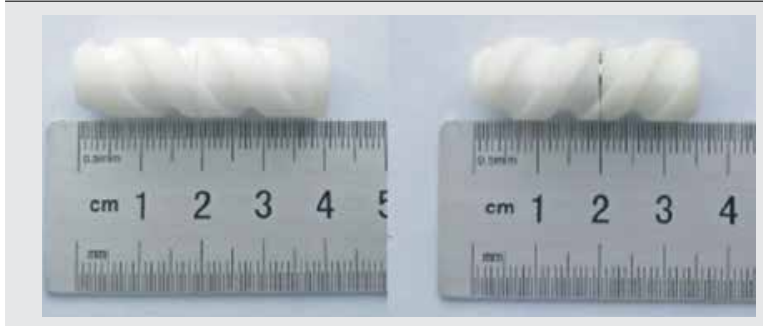

(c) [2017] IEEE Reprinted, with permission, from Guo J, Liu P, Guo S, et al. Development Automation (ICMA), 2017 IEEE International Conference on IEEE 2017; 439-444. of a novel wireless spiral capsule robot with modular structure. Mechatronics and

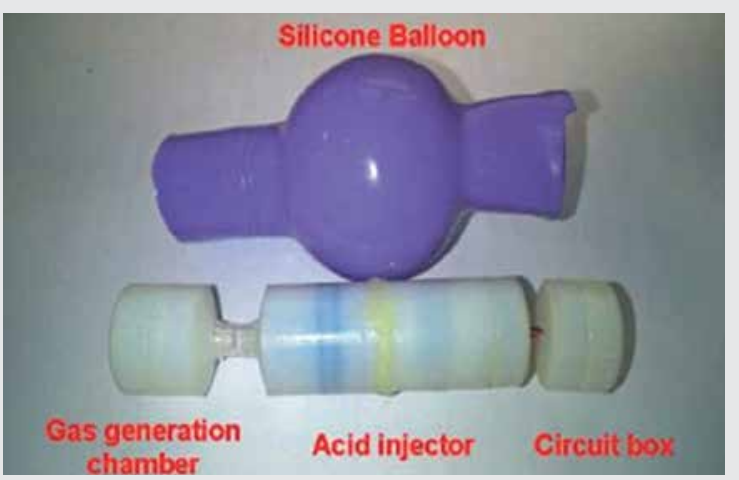

(C) [2017] IEEE Reprinted, with permission, from Leung BHK, Poon CCY, Zhang R, et al. A Therapeutic Wireless Capsule for Treatment of Gastrointestinal Haemorrhage by Balloo Tamponade Effect. IEEE Transactions on Biomedical Engineering 2017;64: 1106-14. Capsule for Haemostasis Utilising an Inflated Balloon [35]

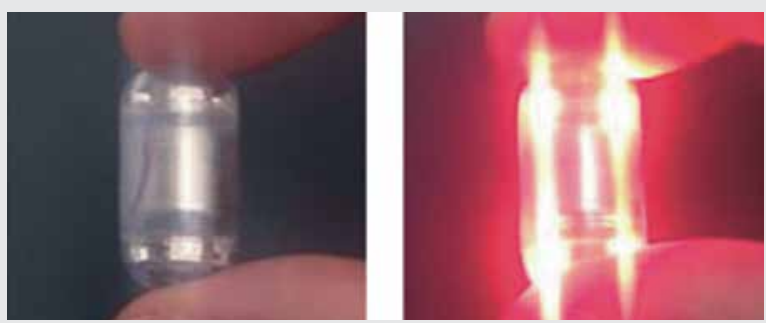

(c) [2016] IEEE Reprinted, with permission, from Tortora G, Orsini B, Pecile P, et al. An ingestible capsule for the photodynamic therapy of helicobacter pylori infection. IEEE/ ASME Transactions on Mechatronics 2016; 21: 1935-42.

Blue and Red Light Capsule for Photodynamic Therapy of Helicobacter Pylori [37]

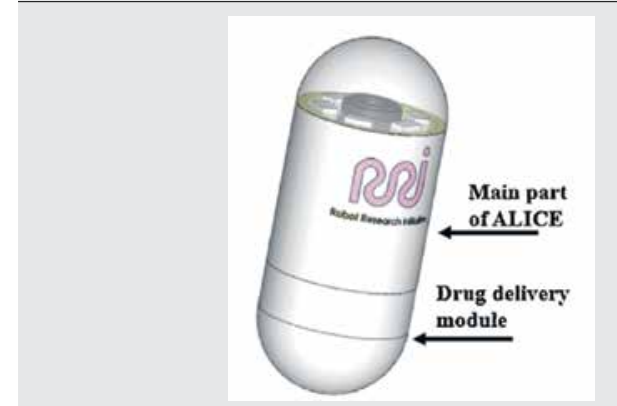

Reprinted from Le VH, Rodriguez HL, Lee C, Go G,et al. A soft-magnet-based drug-delivery module for active locomotive intestinal capsule endoscopy using an electromagnetic actuation system. Sensors and Actuators A Physical 2016; 243: 81-9. Copyright (2016), with permission from Elsevier

A Soft-Magnet-Based Drug-Delivery Module for ALICE System [38]

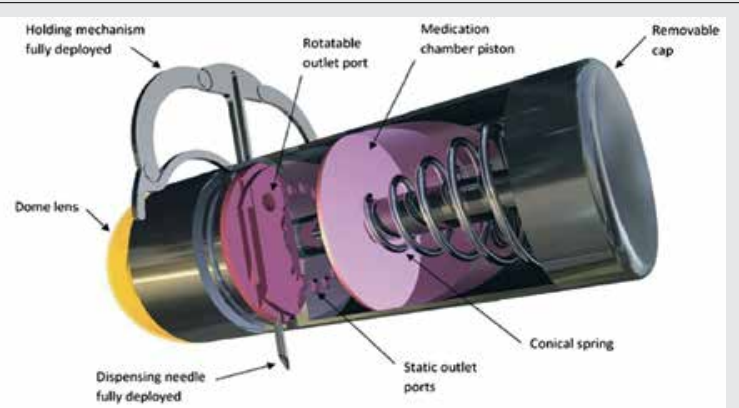

Woods SP, Constandinou TG. A compact targeted drug delivery mechanism for a next generation wireless capsule endoscope. Journal of Micro-bio Robotics, https:// doi.org/10.1007/s12213-016-0088-9. Copyright @ 2016, Woods SP, Constandinou TG, springer Berlin Heidelberg licensed by Creative Commons CC BY license.

A wireless capsule endoscope with holding mechanism for medication release [39]

\section{(1). (14 \\ Fig 5. System packaging. (1RF Antenna, (2) pH Sensor, (2RF Circuit, (9Micro-controller, (1Power} Management. (Batterv. () LED Driver. (B) Blue LEDs. (Shell.

Li Z, Ren B, Tan H, et al. Capsule Design for Blue Light Therapy against Helicobacter pylori. PLoS One 2016; 11: e0147531. https://doi.org/10.1371/journal.pone.0147531 Copyright: @ 2016 Li et al licensed by Creative Commons CC BY license Blue Light Therapy Capsule for Helicobacter pylori [40]

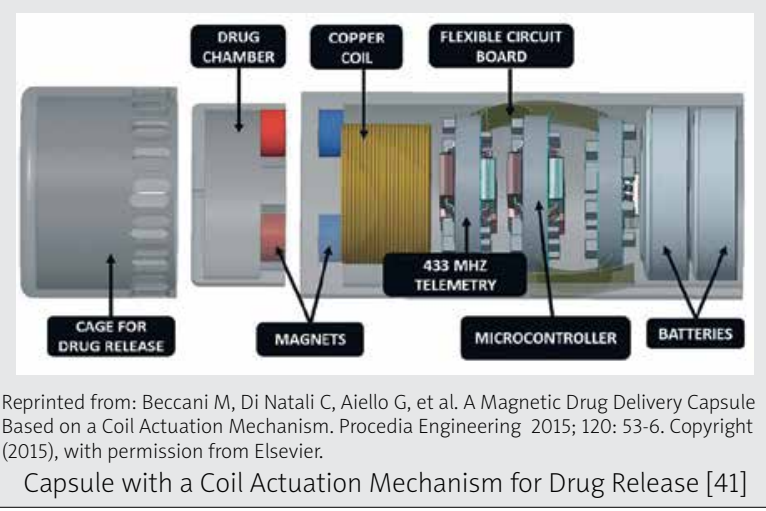


Table II. Cont.

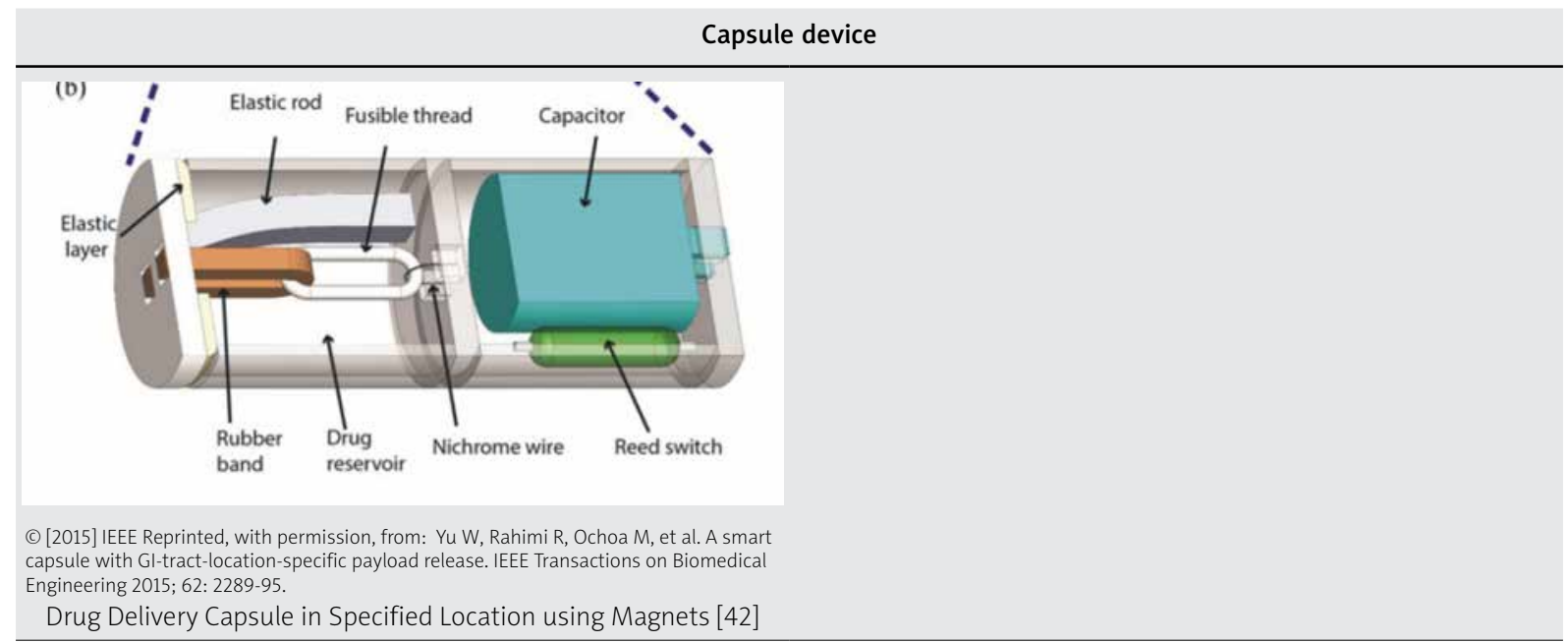

been proposed by the group of Leung et al. [35]. The capsule consists of three segments linked with flexible joints. These segments are a gas generation chamber with a length of $13 \mathrm{~mm}$, an acid injector with a length of $35 \mathrm{~mm}$, and a circuit box with a length of $12 \mathrm{~mm}$. The capsule has a diameter of $14 \mathrm{~mm}$, and it is enclosed in a silicone balloon. The balloon inflates at a bleeding lesion and achieves haemostasis by the tamponade effect. The inflation of the balloon is achieved by an acid injection into a gas generation chamber filled with base powder. The amount of infused acid controls the pressure and the volume of the silicone balloon in order to suit the variation of the diameter and texture of the intestine. The inflation of the balloon is capable of producing appropriate pressure to the bowel wall to anchor the capsule steadily in the position of the bleeding. Ex vivo experiments for the evaluation of the appropriate pressure and in vivo experiments of bleeding in the small intestine of a pig were conducted, showing that the proposed capsule is able to achieve haemorrhage control in the lower Gl.

\section{A wireless spiral capsule robot with modular structure}

Detection and treatment of GI pathologies are two functionalities, which need different hardware integration, and the co-existence of both in the same capsule is limited by its size. Thus, the group of Guo et al. [36] has proposed a wireless spiral capsule robot with modular structure driven by the external magnetic field. The capsule robot consists of two robots with modular structure with a mechanism to combine and separate them. It consists of a guide robot and an auxiliary robot with helical diversion grooves. The guide robot has a length of $39.4 \mathrm{~mm}$ and a diameter of $12.5 \mathrm{~mm}$. The auxiliary robot has a length of $33.8 \mathrm{~mm}$ and a diameter of $12.5 \mathrm{~mm}$. In the two robots, permanent magnets have been placed in the centre and magnetized in the radial direction. Under the same external magnetic field, generated by the three-axis Helmholtz coils, the motion of each capsule is relative to the other. After the inspection of the guide module robot, the treatment module robot can be swallowed and then dock with the guide module robot, thus saving the long time normally required to reposition the target location.

\section{Blue and red light capsule for photodynamic therapy of Helicobacter pylori}

The group of Tortora et al. [37] investigate the efficiency of LEDs in specific wavelengths needed for the therapy of infection from Helicobacter pylori. Based on their measurements, two easy-to-swallow capsule devices have been developed. The first is a capsule for research purposes, which has a more performant battery that is not permitted in clinical examination. The second is a preindustrial capsule that integrates certified modules for clinical examination. The capsules integrate eight LEDs placed in an electronic board along with a magnetic switch and a battery. Two versions of the capsule have been constructed based on the emitting wavelength. One emits red light only at $625 \mathrm{~nm}$ and the other blue light at $405 \mathrm{~nm}$. The capsules have a diameter of $14 \mathrm{~mm}$ and length of $27 \mathrm{~mm}$.

\section{Soft-magnet-based drug-delivery module for the ALICE System}

A module for drug delivery that is combined with the ALICE [25] system has been presented by the group of Le et al. [38]. The drug delivery module consists of 
two ring-type soft magnets and a simple plastic hinge. The ring-type magnets are axially magnetised, attracting each other and keeping the drug enclosed inside the module. The ALICE system provides controlled navigation of the integrated ALICE and drug delivery module to investigate and accurately infuse the drug to the lesion area. The drug-delivery module is opened by the repulsive force between the two radially magnetised soft-magnetic rings, when the axial magnetisation of the rings stops. The rings are demagnetised and a strong pulsating magnetic field in a radial direction is applied, and the drug enclosed in the module is released. Then, the two rings are axially magnetised again, attracting each other, and thanks to the plastic hinge the drug-delivery module is returned to its initial shape. The integrated drug delivery module with ALICE system has a diameter of $12 \mathrm{~mm}$ and a length of $33 \mathrm{~mm}$. The proposed drug delivery module with ALICE system has been tested in vitro.

\section{Wireless capsule endoscope with holding mechanism for medication release}

The group of Woods et al. [39] have presented a concept of a microrobot with a medication release and a drug infusion mechanism for targeted drug delivery. The microrobot has been designed with resistance to natural peristalsis, deploying a holding mechanism enabling the microrobot to localise a pathological area of interest in the GI tract. Then, a needle is positioned in this area and delivers a 1-ml dose of medication. The needle has the ability to be placed in a $360^{\circ}$ scale, while simultaneously maintaining a diametrically opposite relationship with the holding mechanism. This feature guarantees the penetration of the Gl tract wall by the needle. The holding mechanism utilises a single micromotor to open and close two legs. The legs stretch and hold the microrobot in the region of interest inside the Gl tract.

\section{Blue light therapy capsule for Helicobacter pylori}

A blue light emission capsule for the therapy of Helicobacter pylori has been proposed by the group of Li et al. [40]. A module for $\mathrm{pH}$ sensing and measuring is used to differentiate locations and evaluate the digestive function by monitoring the $\mathrm{pH}$ values of the $\mathrm{Gl}$ tract. The optical source of the capsule consists of eight blue LEDs which emit blue light for treatment according to a pre-set range of $\mathrm{pH}$ values. Also, the capsule consists of a low power-consumption microcontroller for the processing of the $\mathrm{pH}$ signal and a wireless communication module for the transmission of the measured
$\mathrm{pH}$ values to an external receiver. The proposed capsule has a diameter of $11.5 \mathrm{~mm}$ and a length of $22 \mathrm{~mm}$.

\section{Capsule with a coil actuation mechanism for drug release}

The group of Beccani et al. [41] has designed a prototype Magnetic Drug Delivery Capsule (MDDC). A coil actuation mechanism is used by the capsule for the release of the drug. The capsule consists of a coil, a magnet, and a drug chamber in which matching magnets are placed. The drug chamber remains attached to the body of the capsule by the generated attraction between the magnets. The coil is able to produce the appropriate force for the magnets to be repulsed. Then, the chamber opens, and the drug is released in the specific area of the GI tract. The diameter of the capsule is $13 \mathrm{~mm}$ and the length is $30 \mathrm{~mm}$. The capsule has a weight of $12 \mathrm{~g}$, and the drug chamber can store to $2.4 \mathrm{ml}$ of drug.

\section{Drug delivery capsule in a specified location using magnets}

A smart capsule for location-specific drug release in the GI tract has been presented by the group of $\mathrm{Yu}$ et al. [42]. The proposed capsule has length of $26 \mathrm{~mm}$ and diameter of $9 \mathrm{~mm}$. The specific location for the release of the drug is determined by an implanted or externally worn permanent magnet. The capsule is activated when it is close to the magnet. Then, a reed switch closes and the capacitor is discharged by a nichrome wire. A nylon fuse melts while the cap is opening and the drug is released. The drug delivery procedure was tested in vitro showing the locomotion of the capsule next to the permanent magnet.

\section{State-of-the-art abnormality detection software}

The diagnostic accuracy of CE depends on the clinician's acumen and attention when examining a large amount of collected images from each examination. Reviewing a CE examination video is a very time-consuming task, which can often be a burden in everyday clinical practice, especially in units with high turnaround of CE procedures. Therefore, intense research activity has been developed aiming to achieve accurate and reliable automated diagnostic software. To date, several efforts have been made towards constructing computer-based medical systems (CBMS) capable of analysing CE image sequences for the detection and recognition of abnormalities. However, establishing the clinical effectiveness and confidence in such diagnostic systems requires further research. 
Usually, CBMS are designed so as to classify CE images into normal or abnormal categories and/or to discriminate sets of pixels, i.e. image segments that represent abnormalities. Such systems can contribute to a reduction in the number of false negative diagnoses. Before classification, the endoscopic images undergo a processing stage facilitating the enhancement of any notable features. These features numerically represent information about the images, such as colour, texture, and shape. Image classification and/or segmentation are based on such features to discriminate different kinds of pathologies, e.g. bleeding, ulcer, polyps, etc.

Usually, supervised classification algorithms, such as Artificial Neural Networks (ANNs), and Support Vector Machines (SVMs) [43] are employed for image classification and/or segmentation. The results are presented in terms of average accuracy (ACC), representing the number of correctly detected abnormal samples divided by the total number of samples, and/or the average sensitivity (SN), which represents the true positive detection rate, and the specificity (SP), which represents the true negative detection rate. In some studies the precision is provided instead of SP, the former representing the proportion of true positives over all positives. Also, in some studies, the Receiver Operating Characteristic (ROC) and the Area Under the ROC (AUC) are used as a more reliable metric for abnormality detection. Interestingly, most of these studies have focused on the detection of one category of pathologies. Only a few of them have focused on the detection of suspicious CE video frames, regardless of the pathology.

The following paragraphs present the state-of-theart abnormality detection methods for computer-based medical software systems, per abnormality type.

\section{Blood detection methods}

Computer-based abnormality detection systems have proved especially useful in detecting and locating the origin of obscure gastrointestinal bleeding (OGIB), which is defined as chronic bleeding from a source not found after traditional (wired) endoscopy. Most of the proposed systems concentrate on features associated with the colour of the image, as blood has a distinct red hue. Colour is the most important aspect that differentiates bleeding and non-bleeding regions. However, in some cases edge pixels, such as those found in intestinal folds, and bleeding pixels share similar dark hues, which means that traditional algorithms often mistake edge pixels for bleeding pixels. Fu et al. [44] and Usman et al. [45] used a technique to remove these edge pixels aiming to enhance bleeding detection. Usman et al. used [45] the transformation of an image to Hue saturation value (HSV) colour space to extract image features, while Fu et al. [44] grouped pixels adaptively into uniformly-coloured segments based on colour and location, with a procedure called superpixel segmentation. The feature used from each superpixel for bleeding detection is the ratio of red to green, blue, or the sum of red, green, and blue intensities in RGB (Red, Green, Blue) colour space.

Yuan et al. [46] considered quantised colour histograms as features for blood detection, using a technique named Bag of Words (BoW). This technique represents each image with a set of "visual" words, corresponding to image segments. In the second stage, the bleeding regions are localised using a saliency map that indicates regions of importance within the image, estimated based on colour information from various colour spaces. A classifier fusion algorithm to detect the bleeding frames and localise the bleeding area was proposed by Deeba et al. [47]. It combines the results of two classifiers trained using first-order statistical features extracted from RGB and HSV colour spaces, which include mean, standard deviation, entropy, skew, and energy. Furthermore, Ghosh et al. [48] noted that the blue (B) component of RGB does not carry any valuable information for the discrimination between bleeding and non-bleeding zones. Instead, a composite colour component obtained by dividing the green $(\mathrm{G})$ with the red $(R)$ components, i.e. $G / R$, was found to be more informative. First-order statistical features were extracted from $G / R$ and were used as the input to an SVM classifier for the detection of bleeding frames.

As well as colour features, texture and shape features have been also considered for the discrimination of bleeding frames. In the study conducted by $\mathrm{Hu}$ et al. [49], a geometric image feature, called local-contrast-enhanced higher-order local auto-correlation (LCEHLAC), was utilised along with an image pre-processing method for a non-linear conversion model of the HSV colour space. A methodology combining colour histogram features and automatically extracted features was proposed by Jia et al. [50]. The former are extracted from CIE-Lab colour space, and the latter are extracted using a deep (multi-layer) neural network architecture, called the Convolutional Neural Network (CNN).

A summary of the aforementioned blood detection methods along with their results in various datasets is provided in Figure 1. It can be noticed that in all cases the performance metrics are high, exceeding $90 \%$. The different methods cannot be directly compared to each other because they have been tested on different datasets. It is notable that the highest overall performance was reported by Hu et al. [49], with an AUC of 99\%, using one of the largest datasets, with 11,118 image frames, as compared to the other reviewed studies. 


\section{Polyp and tumour detection methods}

Polyps are growing protrusions of mucosa inside the intestine due to excessive proliferation of tissue and inflammation or deep-seated malformations. Polyps are mainly discriminated by their shape and texture [49]. Mamonov et al. [51] started by accepting polyps as protrusions that are mostly round in shape. Thus, the best-fit ball radius was used as a decision parameter of a classifier. Yuan et al. [52] use the Scale-Invariant Feature Transform (SIFT) to detect salient points in images that may correspond to polyps. From the neighbourhoods of these points, texture features, called Complete Local Binary Pattern (CLBP), are extracted. A more complex methodology was proposed by Liu et al. [53] for small-bowel tumour detection. This methodology is based on both texture and colour analysis. Multi-scale texture analysis is performed (i.e. the analysis is performed at different image resolutions) by means of the curvelet transformation and fractal encoding. Colour information is captured by means of higher order moments between different colour channels. The extracted features are classified by an optimally selected SVM classifier. In another study, Alizadeh et al. [54] proposed an adaptive neuro-fuzzy inference system aimed at classifying CE video frames containing polyps. The system extracts 32 features including four statistical measures (namely, contrast, correlation, homogeneity, and energy) calculated from co-occurrence matrices. Mutual information (a measure of the information shared between pairs of features) was used to select a subset of more informative features for the discrimination of polyps from normal tissues.

The results of the polyp/tumour detection methods reviewed in this subsection are presented in Figure 1. The highest results were reported by Liu et al. [53], who achieved an accuracy of $97.3 \%$. However, the dataset used is smaller than the one used by Hu et al. [49], who achieved an accuracy of $92.4 \%$. The lower accuracy of the latter can be mainly attributed to its lower specificity.

\section{Ulcer detection methods}

Ulcers are among the most common pathologic outcomes of several diseases affecting the GI tract. Small-bowel ulcers, of variable severity and activity, are often challenging in terms of detection by traditional imaging techniques. Hence, CE is increasingly being used in ulcer diagnosis and management. Several solutions have been proposed for the detection and follow-up of different forms of CD or NSAID-induced ulcerations. In this context, the majority of recent studies were based on combinations of texture and colour fea-

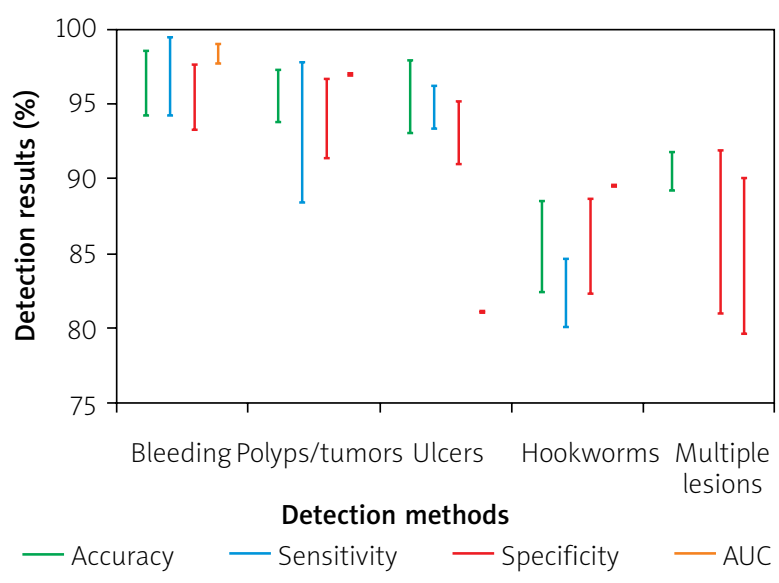

Figure 1. Graphical representation and comparison of the aforementioned detection methods for the different kind of abnormalities during the last 5 years (2014-2018). The vertical bars indicate overall ranges of accuracy, sensitivity and specificity obtained from various abnormality detection methods. Single points indicate no variance. Missing bars indicate unavailable data due to inconsistent reporting of results across studies

tures to discriminate ulcers from normal tissues. In the study of Suman et al. [55], the colour components of seven different colour spaces were analysed in order to find an optimal combination of their colour components that better discriminates ulcers from normal tissues. The selected components were $\mathrm{Cr}$ (which represents the difference of red from a reference value) from $\mathrm{YCb}$ $\mathrm{Cr}$ colour space, the yellow $(\mathrm{Y})$ component from CMYK colour space, and the blue (B) component from RGB. A two-stage, fully-automated, computer-aided detection system is proposed by Yuan et al. [56] to detect ulcers in CE images. In the first stage, the image is segmented using superpixels of different sizes. Colour and texture features are extracted from each superpixel. Then, the extracted features are fused to form a saliency map per image. In the second stage the BoW technique uses the obtained saliency map to better characterise the images. A system capable of using weakly annotated images was proposed by Vasilakakis et al. [57]. Instead of annotating the images in detail, i.e. pixel-by-pixel, the images can be annotated at image level. In this way, a binary semantic label is assigned per image to indicate whether its content is normal or abnormal, e.g. the keyword "abnormal" if the image contains an abnormality. This system offers the convenience to robustly detect which images contain possible ulcers. A weak image annotation method based on a CNN architecture was presented by Georgakopoulos et al. [58]. The CNN receives a single $C E$ image as input to process, and 
it makes the decision regarding the presence of an inflammatory lesion in the input image (i.e. two-neuron output layer).

Figure 1 gathers the results from the previously presented studies, which focus on the ulcer detection. Suman et al. [55] reported the best results compared to the other methods, achieving an accuracy of $97.9 \%$. However, the results cannot be directly compared to the other methods because different datasets were used. In the method of Georgakopoulos et al. [58], where a CNN architecture is utilised, the performance is noticeably high, with an accuracy of $90.2 \%$. Although the dataset in the study of Georgakopoulos et al. [58] consists of weakly annotated images, automatically extracted features of the proposed method are able to characterise the images and provide a classification system independent of handcrafted features and pixel-level image annotation.

\section{Hookworm detection methods}

Hookworms are a leading cause of maternal and child morbidity, and their detection is often a challenging task. An automatic hookworm detection system for CE images was proposed by Wu et al. [59]. The system is based on the basic characteristics of hookworms, which are their tubular body structure, the parallel edges in the shape of their body, and their body colour features. The first step is the processing of the image for the enhancement and the detection of locations with tubular structure. The second step is a parallel region detection method identifying the potential regions having hookworm bodies. The last step for the detection of hookworms is the features based on their intensity, which discriminate the hookworms from different components of the GI tract, i.e. bubbles. A histogram of average intensity is proposed to represent their properties. A deep hookworm detection system based on CNN was proposed by He et al. [60] for CE images. The system consists of two CNN networks, which enable the simultaneous modelling of visual appearances and tubular patterns of hookworms.

The results of the proposed methods are shown in Figure 1 . The experiments were performed in the same dataset for all the aforementioned hookworm detection methods. It can been noticed that the method of He et al. [60] for the hookworm detection achieved higher classification performance than the method of Wu et al. [59] This can be attributed to the better enhancement of the visual pattern of the hookworms achieved by the CNN.

\section{Multiple lesion detection methods}

The detection of different lesions is one of the most challenging tasks in the CE reviewing process. To this end, the majority of research studies focus on the de- tection of one kind of abnormality, probably because it is easier for an algorithm to distinguish one abnormality each time, i.e. bleeding detection is mainly based on the characteristic red hue of blood. Nawarathna et al. proposed [61] a texture analysis method. Different texture features, i.e. LBP, of an image extracted and the distribution of these various texture features is captured by a histogram to characterise the content of each image. An automated technique was proposed [62] for abnormality detection in CE images. Every image is divided into several blocks and from each block the colour information is extracted using a CNN to overcome the drawbacks of handcrafted features. In [63], the SIFT features are extracted from images in HSV colour space to obtain visual words that represent the three lesion categories (bleeding, polyps, ulcer) and the normal images. Then, these four types of visual words are combined together to composite the representative visual words for classifying the CE images. It is known that the semantics of the normal content include mucosal tissues, the hole of the lumen, bubbles, and debris. Thus, an investigation of such a semantic interpretation of the CE images may yield an improvement in lesion detection. In [64], a system for the semantic interpretation of the whole CE content was proposed, in which each semantic content category, i.e. the hole of the lumen it represents a different label. The system consists of a salient point detection algorithm to detect points of interest in weakly annotated images, and it extracts colour features from the area around them. The representation of the CE images is based on the BoW image representation technique. Then, multi-label SVM classifiers are utilised to discriminate the labels that exist in a CE image. The study of [64] was further extended in [65], where a convolutional neural network architecture enabling multi-scale feature extraction (MM-CNN) was proposed to detect the existing labels in the CE images. In [66], a three-phase methodology based on a CNN architecture for automatic detection and localisation of lesions in CE images was presented. In the first phase, the proposed CNN architecture automatically extracts features from weakly annotated images. In the second phase, it suggests the possible locations of lesions in the detected CE images. In the third phase, a new algorithm is proposed to localise the lesions in the detected CE images. This algorithm uses the automatically extracted features from CNN. Then, the algorithm detects the suggested locations from the second phase, which belong to lesions.

A summary of previously presented methods for the detection of different kinds abnormalities is in Figure 1. It can be noticed that the method proposed by Vasilakakis et al. [65] has considerably higher results, with 
an AUC of $90.0 \%$, which indicates that the semantic interpretation of the content of CE images can provide valuable information to assist in the detection of different lesions.

\section{Conclusions}

The constant improvement of DY in CE over the last few years is noticeable. Automatic lesion detection and interpretation remains one of the niche developmental areas in CE, although several research groups are working on manufacturing capsule endoscopes. This constant improvement of DY prepares the way for research targeting TCE. Potentials of TCE such as drug delivery, haemostasis of the bleeding area, and light therapy of $H$. pylori have been described. The key points of this review study can be summarised as follows:

- Different kinds of capsule endoscopes have been proposed as solutions for image quality enhancement, navigation, biopsy, and wireless power supply.

- Therapeutic capsule endoscopes targeting specific lesions have been investigated.

- There is a clinical need for therapeutic capsule endoscopes for the therapy of pathologies such as gastrointestinal bleeding, Crohn's disease, and small-intestine tumours.

- Most capsule endoscopes intend to improve the examination and therapy of the whole gastrointestinal tract.

- Different drug release and delivery mechanisms for capsule endoscopes have been presented in this review.

- The advances of capsule endoscopes in control and navigation inside the GI tract contributes to the progress of capsule endoscopes targeting the treatment.

- Most of the capsule endoscopes are concepts or prototypes without approval for in vivo clinical experiments.

- Some capsule endoscopes have diameter and/or length that make swallowing difficult.

- At this time there is no commercial or research capsule endoscope with all the aforementioned functionalities.

- The development of high-resolution, high-definition images should be seen as the cornerstone for further active promotion and wider clinical adoption of capsules.

- Automatic lesion detection and reporting and development of an accurate lesion localisation systems remain a priority software challenge.

- The establishment of standard, sufficiently large datasets for CE for lesion detection experiments is necessary.
In the future, CE systems should be used as multifunctional units capable of both diagnosis and treatment at specified sites of the Gl tract following the instructions from physicians. Despite the fact that the number of proposed methods is continuously increasing, the actual progress with respect to CE software can only be considered marginal. The main drawbacks for essential progress of lesion diagnosis and problems constraining the entry of therapeutic capsules in the market can be summarised as follows:

- The lesion detection software lacks public, large, and diverse datasets.

- The lesion detection methods that were presented have to evaluate comparatively using a standard database of CE images/videos.

- The lack of regional controllability over drug delivery in the target areas, in order to prevent the spreading of medication over other areas of Gl tract.

- Treatment capsule endoscopes must be able to navigate either internally or externally and anchor at the target for therapy area inside the GI tract.

- Insufficient power backup.

- The size of the aforementioned experimental capsules has to be adjusted, in order to ease swallowing for the examination and/or treatment of the patient.

- Miniaturisation of the drug chamber, in order to facilitate the ingestibility of therapeutic capsules due to their limited size.

\section{Acknowledgments}

This work was supported in part by the project "Klearchos Koulaouzidis", Grant No. 5151, and Grant No. 5024 of the Special Account of Research Grants of the University of Thessaly, Greece.

\section{Conflict of interest}

The authors declare no conflict of interest.

\section{References}

1. lakovidis DK, Koulaouzidis A. Software for enhanced video capsule endoscopy: challenges for essential progress. Nature Rev Gastroenterol Hepatol 2015; 12: 172-86.

2. Spada C, McNamara D, Despott EJ, et al. Performance measures for small-bowel endoscopy: a European Society of Gastrointestinal Endoscopy (ESGE) Quality Improvement Initiative. Endoscopy 2019; 51: 574-98.

3. PillCam ${ }^{\mathrm{TM}}$ Capsule Endoscopy User Manual PillCam ${ }^{\mathrm{TM}}$ Desktop Software Version 9.0 DOC-2928-02 November 2016.

4. Available from: http://www.intromedic.com/eng/main/.

5. Available from: https://www.olympus-europa.com/medical/ en/Products-and-Solutions/Products/Product/ENDOCAPSULE-10-System.html. 
6. Available from: http://www.capsovision.com/physicians/product-specifications.

7. Available from: http://english.jinshangroup.com/capsuleendoscopy.html.

8. Available from: https://www.check-cap.com/.

9. Yung DE, Koulaouzidis A, Avni T, et al. Clinical outcomes of neg ative small-bowel capsule endoscopy for small-bowel bleeding: a systematic review and meta-analysis. Gastrointest Endosc 2017; 85: 305-17.

10. Sonnenberg A. Modeling lengthy work-ups in gastrointestinal bleeding. Clin Gastroenterol Hepatol 2015; 13: 433-9.

11. Woodward Z, Williams JL, Sonnenberg A. Length of endoscopic workup in gastrointestinal bleeding. Eur J Gastroenterol Hepatol 2016; 28: 1166-71

12. Available from: https://clinicaltrials.gov/ct2/results?cond=Capsule+endoscopy\&term $=$ Capsule+endoscopy \&cntry $=\&$ state $=\&$ city $=\&$ dist $=$

13. Fisher LR, Hasler WL. New vision in video capsule endoscopy: current status and future directions. Nature Rev Gastroenterol Hepatol 2012; 9: 392-405.

14. Koulaouzidis A, Douglas S. Capsule endoscopy in clinical practice: concise up-to-date overview. Clin Exp Gastroenterol 2009, 2: 111-6.

15. Koulaouzidis A, lakovidis DK, Karargyris A, et al. Optimizing lesion detection in small-bowel capsule endoscopy: from present problems to future solutions. Exp Rev Gastroenterol Hepatol 2015; 9: 217-35.

16. Mapara SS, Patravale VB. Medical capsule robots: a renaissance for diagnostics, drug delivery and surgical treatment. J Control Release 2017; 261: 337-51.

17. Vasilakakis M, Koulaouzidis A, Yung DE, et al. Follow-up on: optimizing lesion detection in small-bowel capsule endoscopy and beyond: from present problems to future solutions. Expert Rev Gastroenterol Hepatol 2019; 13: 129-41.

18. Jang J, Lee J, Lee KR, et al. 4-Camera VGA-resolution capsule endoscope with $80 \mathrm{Mb} / \mathrm{s}$ body-channel communication transceiver and Sub-cm range capsule localization. Solid-State Circuits Conference-(ISSCC), 2018 IEEE International. IEEE 2018; 282-4

19. Fontana R, Mulana F, Cavallotti C, et al. An innovative wireless endoscopic capsule with spherical shape. IEEE Trans Biomed Circ Systems 2017; 11: 143-52.

20. Son D, Dogan MD, Sitti M. Magnetically actuated soft capsule endoscope for fine-needle aspiration biopsy. Robotics and Automation (ICRA), 2017 IEEE International Conference on. IEEE; 2017; 1132-9.

21. Fu Q, Guo S, Guo J. Conceptual design of a novel magnetically actuated hybrid microrobot. Mechatronics and Automation (ICMA), 2017 IEEE International Conference on. IEEE; 2017; 1001-5.

22. Winstone B, Melhuish C, Pipe T, et al. Toward bio-inspired tactile sensing capsule endoscopy for detection of submucosa tumors. IEEE Sensors J 2017; 17: 848-57.

23. Demosthenous P, Pitris C, Georgiou J. Infrared fluorescence-based cancer screening capsule for the small intestine. IEEE Trans Biomed Circ Syst 2016; 10: 467-76.

24. Gao J, Yan G, Wang Z, et al. Design and testing of a motor-based capsule robot powered by wireless power transmission. IEEE/ASME Transact Mechatron 2016; 21: 683-93.
25. Lee C, Choi H, Go G, et al. Active locomotive intestinal capsule endoscope (ALICE) system: a prospective feasibility study. IEEE/ASME Transact Mechatron 2015; 20: 2067-74.

26. Gu Y, Xie X, Li G, et al. Design of endoscopic capsule with multiple cameras. IEEE Trans Biomed Circ Systems 2015; 9: 590-602.

27. Zhong Y, Du R, Chiu PW. Tadpole endoscope: a wireless micro robot fish for examining the entire gastrointestinal (GI) tract. HKIE Transactions 2015; 22: 117-22.

28. Shi Y, Yan G, Chen W, et al. Micro-intestinal robot with wireless power transmission: Design, analysis and experiment. Computers in Biology and Medicine 2015; 66: 343-51.

29. Yim S, Gultepe E, Gracias DH, et al. Biopsy using a magnetic capsule endoscope carrying, releasing, and retrieving untethered microgrippers. IEEE Trans Biomed Engineering 2014; 61: 513-21.

30. Yim S, Jeon D. Magnetic mechanical capsule robot for multiple locomotion mechanisms. Int J Control Automation Systems 2014; 12: 383-9.

31. Yim S, Jeon D. Capsular microrobot using directional friction spiral. 2009 IEEE International Conference on Robotics and Automation. IEEE 2009; 4444-9.

32. Sun ZJ, Ye B, Qiu Y, et al. Preliminary study of a legged capsule robot actuated wirelessly by magnetic torque. IEEE Trans Magnet 2014; 50: 1-6.

33. Chen WW, Yan GZ, Liu H, et al. Design of micro biopsy device for wireless autonomous endoscope. Int J Precision Engineering Manufacturing 2014; 15: 2317-25.

34. Stewart F, Newton IP, Näthke I, et al. Development of a therapeutic capsule endoscope for treatment in the gastrointestinal tract: Bench testing to translational trial. Ultrasonics Symposium (IUS), 2017 IEEE International. IEEE 2017; 1-4.

35. Leung BHK, Poon CCY, Zhang R, et al. A therapeutic wireless capsule for treatment of gastrointestinal haemorrhage by balIoon tamponade effect. IEEE Trans Biomed Engineering 2017; 64: 1106-14.

36. Guo J, Liu P, Guo S, et al. Development of a novel wireless spiral capsule robot with modular structure. Mechatronics and Automation (ICMA), 2017 IEEE International Conference on. IEEE 2017; 439-44.

37. Tortora G, Orsini B, Pecile P, et al. An ingestible capsule for the photodynamic therapy of helicobacter pylori infection. IEEE/ ASME Transact Mechatron 2016; 21: 1935-42.

38. Le VH, Rodriguez HL, Lee C, et al. A soft-magnet-based drug-delivery module for active locomotive intestinal capsule endoscopy using an electromagnetic actuation system. Sensors Actuators A Physical 2016; 243: 81-9.

39. Woods SP, Constandinou TG. A compact targeted drug delivery mechanism for a next generation wireless capsule endoscope. J Microbio Robotics 2016; 11: 19-34.

40. Li Z, Ren B, Tan H, et al. Capsule design for blue light therapy against Helicobacter pylori. PLoS One 2016; 11: e0147531.

41. Beccani M, Di Natali C, Aiello G, et al. A magnetic drug delivery capsule based on a coil actuation mechanism. Procedia Engineering 2015; 120: 53-6.

42. Yu W, Rahimi R, Ochoa M, et al. A smart capsule with GItract-location-specific payload release. IEEE Trans Biomed Engineering 2015; 62: 2289-95. 
43. Theodoridis S, Koutroumbas K. Pattern Recognition. $4^{\text {th }}$ edn. Academic Press Inc., Orlando, FL, USA 2008.

44. Fu Y, Zhang W, Mandal M, et al. Computer-aided bleeding detection in WCE video. IEEE J Biomed Health Informatics 2014; 18: 636-42.

45. Usman MA, Satrya GB, Usman MR, et al. Detection of small colon bleeding in wireless capsule endoscopy videos. Comp Med Imaging Graphics 2016; 54: 16-26.

46. Yuan Y, Li B, Meng MQ. Bleeding frame and region detection in the wireless capsule endoscopy video. IEEE J Biomed Health Inform 2016; 20: 624-30.

47. Deeba F, Islam M, Bui FM, et al. Performance assessment of a bleeding detection algorithm for endoscopic video based on classifier fusion method and exhaustive feature selection. Biomed Signal Proces Control 2018; 40: 415-24.

48. Ghosh T, Fattah SA, Wahid KA. Automatic computer aided bleeding detection scheme for wireless capsule endoscopy (WCE) video based on higher and lower order statistical features in a composite color. J Med Biol Engin 2018; 38: 482-96.

49. Hu E, Sakanashi $\mathrm{H}$, Nosato $\mathrm{H}$, et al. Bleeding and tumor detection for capsule endoscopy images using improved geometric feature. J Med Biol Engin 2016; 36: 344-56.

50. Jia X, Meng MQ. Gastrointestinal bleeding detection in wireless capsule endoscopy images using handcrafted and CNN features. Proceedings of the Annual International Conference of the IEEE Engineering in Medicine and Biology Society, EMBS 2017; 3154-7.

51. Mamonov AV, Figueiredo IN, Figueiredo PN, et al. Automated polyp detection in colon capsule endoscopy. IEEE Trans Med Imaging 2014; 33: 1488-502.

52. Yuan Y, Li B, Meng MQ. Improved bag of feature for automatic polyp detection in wireless capsule endoscopy images. IEEE Trans Automation Sci Engineering 2016; 13: 529-35.

53. Liu G, Yan G, Kuang S, et al. Detection of small bowel tumor based on multi-scale curvelet analysis and fractal technology in capsule endoscopy. Comp Biol Med 2016; 70: 131-8.

54. Alizadeh M, Maghsoudi OH, Sharzehi K, et al. Detection of small bowel tumor in wireless capsule endoscopy images using an adaptive neuro-fuzzy inference system. J Biomed Res 2017; 31: 419-27.

55. Suman S, Hussin FA, Malik AS, et al. Feature selection and classification of ulcerated lesions using statistical analysis for WCE images. Appl Sci (Switzerland) 2017; 7.

56. Yuan Y, Wang J, Li B, et al. Saliency based ulcer detection for wireless capsule endoscopy diagnosis. IEEE Trans Med Imaging 2015; 34: 2046-57.

57. Vasilakakis M, lakovidis DK, Spyrou E, et al. Weakly-supervised lesion detection in video capsule endoscopy based on a bagof-colour features model. 2017; 96-103.

58. Georgakopoulos SV, lakovidis DK, Vasilakakis M, et al. Weakly-supervised convolutional learning for detection of inflammatory gastrointestinal lesions. IST 2016 - 2016 IEEE International Conference on Imaging Systems and Techniques, Proceedings 2016; 510-4.

59. Wu X, Chen H, Gan T, et al. Automatic hookworm detection in wireless capsule endoscopy images. IEEE Trans Med Imaging 2016; 35: 1741-52.
60. He J, Wu X, Jiang Y, et al. Hookworm detection in wireless capsule endoscopy images with deep learning. IEEE Trans Image Processing 2018; 27: 2379-92.

61. Nawarathna R, Oh J, Muthukudage J, et al. Abnormal image detection in endoscopy videos using a filter bank and local binary patterns. Neurocomputing 2014; 144: 70-91.

62. Sekuboyina AK, Devarakonda ST, Seelamantula CS. A convolutional neural network approach for abnormality detection in Wireless Capsule Endoscopy. Proceedings - International Symposium on Biomedical Imaging 2017; 1057-60.

63. Yuan Y, Li B, Meng MQ. WCE abnormality detection based on saliency and adaptive locality-constrained linear coding. IEEE Trans Automation Sci Engineering 2017; 14: 149-59.

64. Vasilakakis MD, lakovidis DK, Spyrou E, et al. Beyond lesion detection: towards semantic interpretation of endoscopy videos. Engineering Applications of Neural Networks: $18^{\text {th }}$ International Conference, EANN 2017, Athens, Greece, August 25-27, 2017, Proceedings 2017; 379-90.

65. Vasilakakis MD, Diamantis D, Spyrou E, et al. Weakly supervised multilabel classification for semantic interpretation of endoscopy video frames. Evolving Systems 2018; 1-13.

66. Iakovidis DK, Georgakopoulos SV, Vasilakakis M, et al. Detecting and locating gastrointestinal anomalies using deep learning and iterative cluster unification. IEEE Trans Med Imaging 2018; 37: 2196-210.

Received: 11.06 .2019

Accepted: 17.06 .2019 